\title{
Radionuclide Metrology and Standards in Nuclear Physics
}

\author{
Patrick H. Regan ${ }^{1,2}$, Steven M. Judge ${ }^{3}$, John D. Keightley ${ }^{1}$ and Andy K. Pearce ${ }^{1}$ \\ ${ }^{1}$ Chemical, Medical and Environmental Science Division, National Physical \\ Laboratory, Teddington, Middlesex, TW11 OLW, UK \\ ${ }^{2}$ Department of Physics, University of Surrey, Guildford, GU2 7XH, UK \\ ${ }^{3}$ Bureau International des Poids et Mesures, 92312 Sèvres Cedex, France
}

The development of radionuclide standards for metrology has underpinned nuclear physics since its inception [1]. The current frontier of radionuclide metrology relies on developments in radiation detection and signal processing combined with accurate nuclear decay data evaluations [2] and contributes to a myriad of scientific disciplines. Radionuclide metrology represents a crucial part of the scientific jigsaw which enables societal benefits from nuclear physics research.

Measurements that are traceable to internationally accepted primary standards can give the public confidence in the characterization of civilian nuclear waste materials such as ${ }^{90} \mathrm{Sr},{ }^{134,135,137} \mathrm{Cs},{ }^{237} \mathrm{~Np},{ }^{239,240} \mathrm{Pu}$ and ${ }^{241} \mathrm{Am}$ and measurements of naturally occurring radioactive materials (NORMs) such as ${ }^{3} \mathrm{H},{ }^{7} \mathrm{Be},{ }^{14} \mathrm{C},{ }^{210} \mathrm{Po},{ }^{210} \mathrm{~Pb}$,

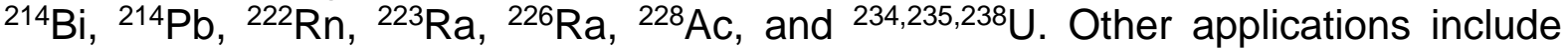
assay of Technologically Enhanced NORM with potential radiological impact on workers in the oil and mineral production industries, and the use of radiopharmaceutical isotopes such as ${ }^{18} \mathrm{~F},{ }^{82} \mathrm{Rb} /{ }^{82} \mathrm{Sr},{ }^{89} \mathrm{Zr},{ }^{99 \mathrm{~m}} \mathrm{Tc},{ }^{124,131} \mathrm{I},{ }^{211} \mathrm{At},{ }^{223} \mathrm{Ra}$ and ${ }^{227}$ Th for diagnostic imaging and therapy. This article explains the concept of international traceability and how accurate radiation standards are determined for different radioactive decay modes.

\section{Primary Radioactivity Standards}

National Measurement Institutes (NMIs) are responsible for the development and upkeep of primary measurement standards. Primary standards are used to calibrate instruments and/or to certify reference materials; these can then be distributed to other laboratories and used to calibrate their own instruments in an uninterrupted chain of calibrations to the final end-user. All measurements are essentially ratios back to these primary standards. NMls cross-check their primary standards against sources from other countries through international comparison exercises co-ordinated by the Bureau International des Poids et Mesures (BIPM).

The first primary standard of radioactivity was based on radium. The inaugural Radium Standards Committee was held in Brussels in 1910 and chaired by Lord Rutherford at which 1 curie $(\mathrm{Ci})$ was defined as the amount of radon in equilibrium with $1 \mathrm{~g}$ of radium [1]. The radioactivity measurement system based on radium standards became outdated following developments in accelerator technology which led to an increased range of artificially created radionuclides. In 1950, the curie was redefined as $3.7 \times 10^{10}$ disintegrations per second and, in 1975, the 15th Conférence Générale des Poids et Mesures adopted the becquerel $(\mathrm{Bq})$ which is equal to one inverse second for the SI unit of activity [3,4]. 
In 1958, The International Committee for Weights and Measures (CIPM) of the BIPM created the Comité Consultatif des Rayonnements lonisants (CCRI)). The CCRI is responsible for organizing international comparisons, enabling NMls to cross-check their primary standards. The International Reference System (SIR), implemented in 1975 , is based on a pressurized well-type ionization chamber, based at BIPM [4]. This is a permanent, stable measurement instrument tool which is available to NMls to compare primary standards of gamma emitters.

A primary standard of radioactivity allows the number of decays from a source in a finite time period to be determined using a technique which does not itself need calibration. Since the activity of each radionuclide species depends on unique decay properties, different experimental techniques are needed for the primary standardisations of individual radioisotopes. The particular technique depends on the radioactive decay mode(s), half-life, decay scheme of the daughter nucleus and branching ratios for competing decay modes. Most modern radioactivity standards are aqueous solutions, quantified by their activity per unit mass on a given reference date $[3,4]$. The main methodologies used for primary radionuclide standardization are discussed below.

\section{High-Geometry Methods}

Perhaps the simplest method is to count the number of photons or particles emitted by a source into $4 \pi$ steradians of solid angle [3]. The perfect $4 \pi$ detector does not exist and the 'non-detection' probability must be accounted for, either by examining the rate of coincidences between multiple detectors or using Monte-Carlo particle transport simulation codes.

For a complex decay scheme of excited states populated in the daughter nucleus, the $4 \pi \gamma$ counting technique can be exploited using either a single well-type $\mathrm{Nal}(\mathrm{TI})$ or two $\mathrm{Nal}(\mathrm{TI})$ detectors sandwiching the source. A higher number of coincident cascade gammas emitted per decay leads to reduced uncertainties from nondetection. Internal conversion transitions can also play a role in increasing the counting efficiency since any associated $\mathrm{x}$-ray emissions can contribute to the integral count rate. Excited levels of the daughter with intrinsic half-lives of the order of the detector dead-time may lead to random summing of a single decay transition which requires appropriate corrections. The gamma-ray counting efficiency may be complemented by a high-efficiency charged particle detector surrounding the source and placed within the well of the gamma detector. Since both detectors may register counts from the same decay event the coincident signals must be subtracted to avoiding double counting; this arrangement is known as $4 \pi \beta+4 \pi \gamma$ counting.

Liquid Scintillation Counting (LSC) techniques use samples mixed with an organic solvent which produces ultraviolet photons following excitation by ionising radiation $[5,6,7]$. These are detected using a photomultiplier tube or a silicon photodiode array. In the efficiency tracing technique, a model of the response of the detector is used, normalised using a tritium standard, which due to its low $Q_{\beta}$ - value $(18 \mathrm{keV})$ provides a reproducible quantification of the low-energy counting losses. The Triple-to-Double-Coincidence Ratio (TDCR) technique overcomes the requirement for a standard through the use of a counting system with three photomultiplier tubes. The model is normalised by matching the measured and modelled ratio of triple to double coincidences. 
In both techniques, the efficiency $(\varepsilon)$ is determined from the non-detection efficiency in each photomultiplier tube using a model based on Poisson statistics. Here $v$ is the energy deposited per decay divided by the energy required to produce a countable pulse, such that,

$$
\varepsilon=1-p(0)=1-\exp (-v)
$$

Since this energy is typically $\sim 1 \mathrm{keV}$ for radioactivities which can deposit hundreds of $\mathrm{keV}$ or more per decay, the non-detection efficiency is virtually negligible. These techniques are particularly useful for radionuclides with no clear gamma-ray decay branch, including 'pure' beta emitters such as ${ }^{3} \mathrm{H},{ }^{14} \mathrm{C},{ }^{32} \mathrm{P}$ and ${ }^{90} \mathrm{Sr}$.

\section{Defined Solid Angle Counting}

Defined solid angle counting is particularly useful for the standardization of alpha-emitting radionuclides and relies on the isotropic emission from a point-like source. In a vacuum, charged particles travel along a straight line through a collimating diaphragm covering a detector with assumed $100 \%$ intrinsic detection efficiency [8]. The system includes baffles to absorb alpha particles emitted in other directions. Only the fraction of the radiation emitted into the solid angle, $\Omega$, subtended by the diaphragm is counted. The observed count rate is corrected for the geometric factor $\Omega / 4 \pi$ as well as for dead-time and decay half-life effects. To minimise counting losses related to source self-absorption, particular care is taken in the preparation of thin, homogenous sources on flat substrates. Typical source-diaphragm distances of 20$40 \mathrm{~cm}$ are used to restrict the counting to particles emitted in a well-defined region perpendicular to the source plane. Suitable radioactive solutions can be standardised with uncertainties of $\sim 0.1 \%$ in the activity concentration value.

\section{Coincidence Counting}

Coincidence counting uses two or more detectors, where each detector is sensitive only to one type of radiation [3,9]. A typical setup involves a charged particle detector such as proportional or scintillation counter situated as close as possible to a $\gamma$-ray detector (e.g. $\mathrm{Nal}(\mathrm{Tl}), \mathrm{LaBr}_{3}$ or $\mathrm{HPGe}$ ). The individual detector count rates are measured together with the coincidence rate within a finite coincidence resolving time and are corrected for detector and electronic dead-times, detector backgrounds and accidental coincidences between separate decays.

In the idealised case of $\beta$-decay to a single excited level of the daughter nuclide which de-excites $100 \%$ via prompt $\gamma$-ray emission, if the count rates of the $\beta$ and $\gamma$-ray detection channels are $\mathrm{N}_{\beta}$ and $\mathrm{N}_{\gamma}$ respectively, and $\mathrm{N}_{\mathrm{c}}$ is the coincidence counting rate then:

$$
\mathrm{N}_{\beta}=A \varepsilon_{\beta} ; \mathrm{N}_{\gamma}=A \varepsilon_{\gamma} ; \text { and } \mathrm{N}_{\mathrm{c}}=\mathrm{A} \varepsilon_{\beta} \varepsilon_{\gamma}
$$

where $\mathrm{A}$ is the unknown source activity and $\varepsilon_{\beta}$ and $\varepsilon_{\gamma}$ are the absolute detection probabilities for the $\beta$ and $\gamma$ channels respectively. These equations combine to give

$$
\mathrm{A}=\mathrm{N}_{\beta} \cdot \mathrm{N}_{\gamma} / \mathrm{N}_{\mathrm{c}} ; \varepsilon \beta=\mathrm{N}_{\mathrm{c}} / \mathrm{N}_{\gamma} ; \varepsilon_{\gamma}=\mathrm{N}_{\mathrm{c}} / \mathrm{N}_{\beta}
$$


These expressions assume that the $\beta$ and $\gamma$-ray detectors are sensitive to $\beta$ particles and $\gamma$ rays exclusively, however, 'non detection' of the $\beta$ particle leaves that detector available to count $\gamma$ rays and/or other ionizing radiations, such as internal conversion and Auger electrons. An additional term $\varepsilon_{\beta \gamma}$ describes this global additional efficiency, yielding the modified expression

$$
A=N_{\beta} /\left[\varepsilon_{\beta}+\left(1-\varepsilon_{\beta}\right) \cdot \varepsilon_{\beta \gamma}\right]
$$

Estimates of the $\beta$ channel counting efficiency may be formed using the measured ratio $\mathrm{N}_{c} / \mathrm{N}_{\gamma}$, together with the apparent activity at a particular effective detection efficiency, $\mathrm{N}_{\beta} . \mathrm{N}_{\gamma} / \mathrm{N}_{\mathrm{c}}$. The expressions are modified to account for multiple $\beta$-decay branches and, if required, competing electron capture decays, internal conversion and Auger electrons, characteristic x-rays etc. The absolute activity $A$ is obtained from the extrapolation of a fit to unit $\beta$ efficiency, see Figure 1.

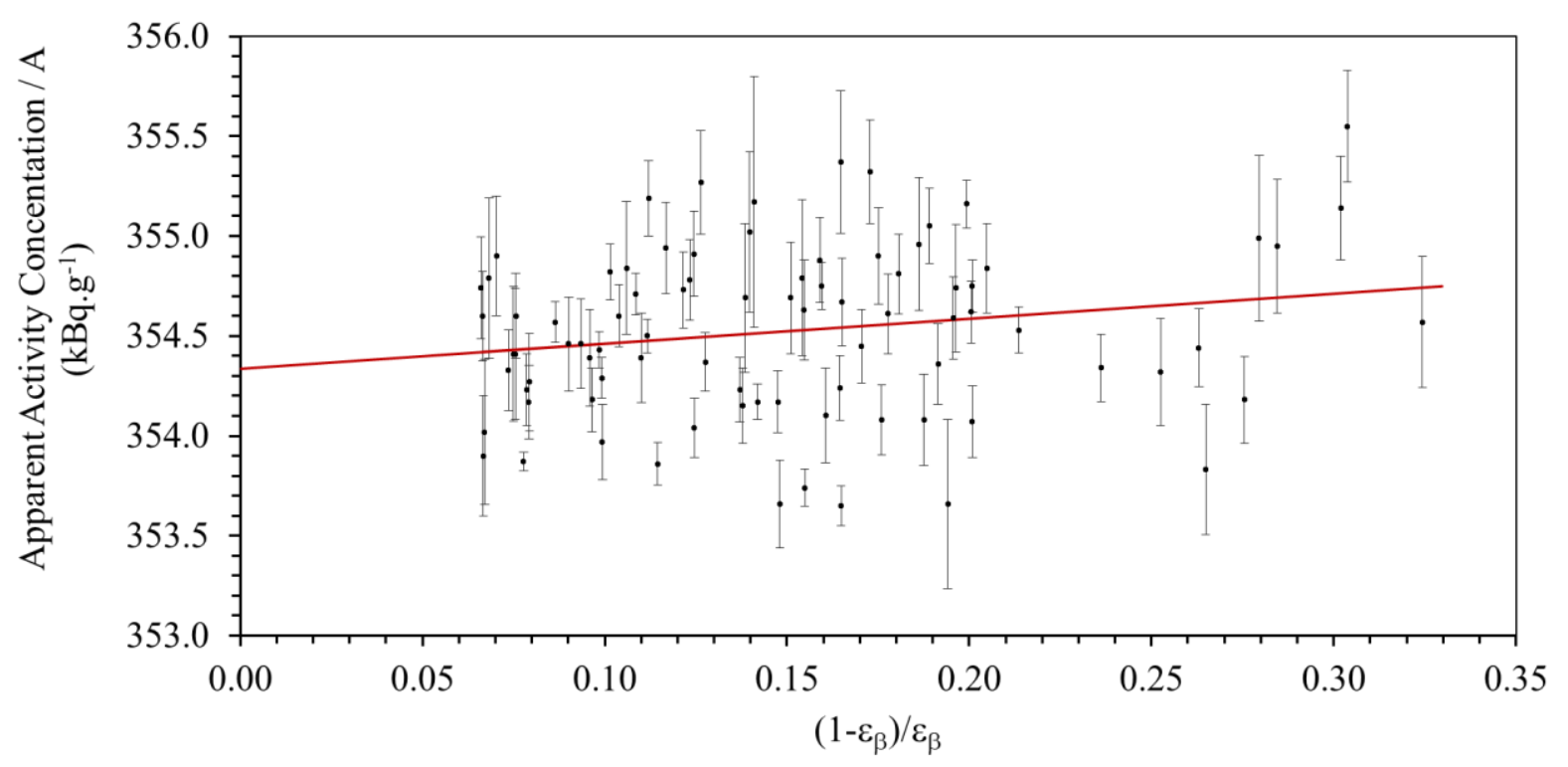

Figure 1: An efficiency extrapolation fit used in the standardisation of $a^{60} \mathrm{Co}$ based on the gamma full-energy peak coincidences with the $4 \pi$ proportional counter [9]. 

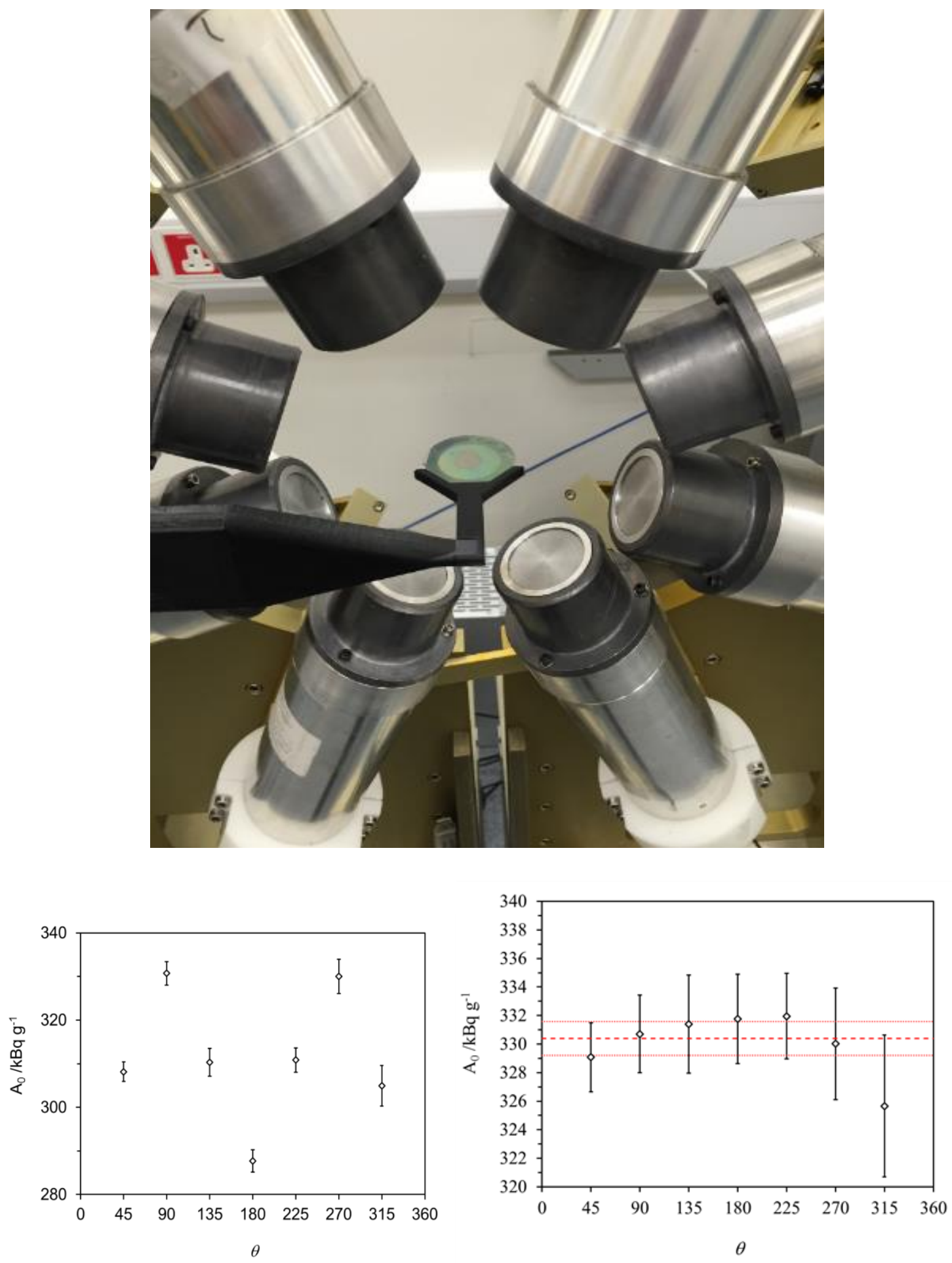

Figure 2: Upper: The UK National Nuclear Array (NANA) which can be used for primary standardisations using the gamma-ray coincidence method; Lower: example of raw and angular correlation corrected primary activity values for $a^{60}$ Co standard taken using NANA [10].

Coincidences between multiple coincident cascade gamma-ray transitions can also be used to determine absolute activity values for decay modes where the coincidence level-scheme in the daughter nucleus is well known, such as the decay of ${ }^{60} \mathrm{Co}$ [10]. The National Nuclear Array (NANA) is a coincident gamma-ray spectrometer based at 
NPL which uses a multi-detector array of $\operatorname{LaBr}_{3}(\mathrm{Ce})$ detectors to allow such measurements, including corrections for angular correlation effects.

\section{Transfer Instruments.}

Most standardisation laboratories maintain a suite of transfer instruments such as ionization chambers calibrated with material standardized using primary techniques. In a re-entrant gas ionization chamber samples are inserted in to a well at the top of the device (see figure 3). The current produced is measured and converted via a calibration factor to an activity for that radionuclide and sample geometry. Since the manufacturing process for ionization chambers is reproducible and the response is steady over decades, calibration factors measured on a master chamber can be applied to replicants. For this reason, ionization chambers are commonly used in hospitals to check radiopharmaceuticals prior to administration to patients and also provide very accurate decay half-life data [10].
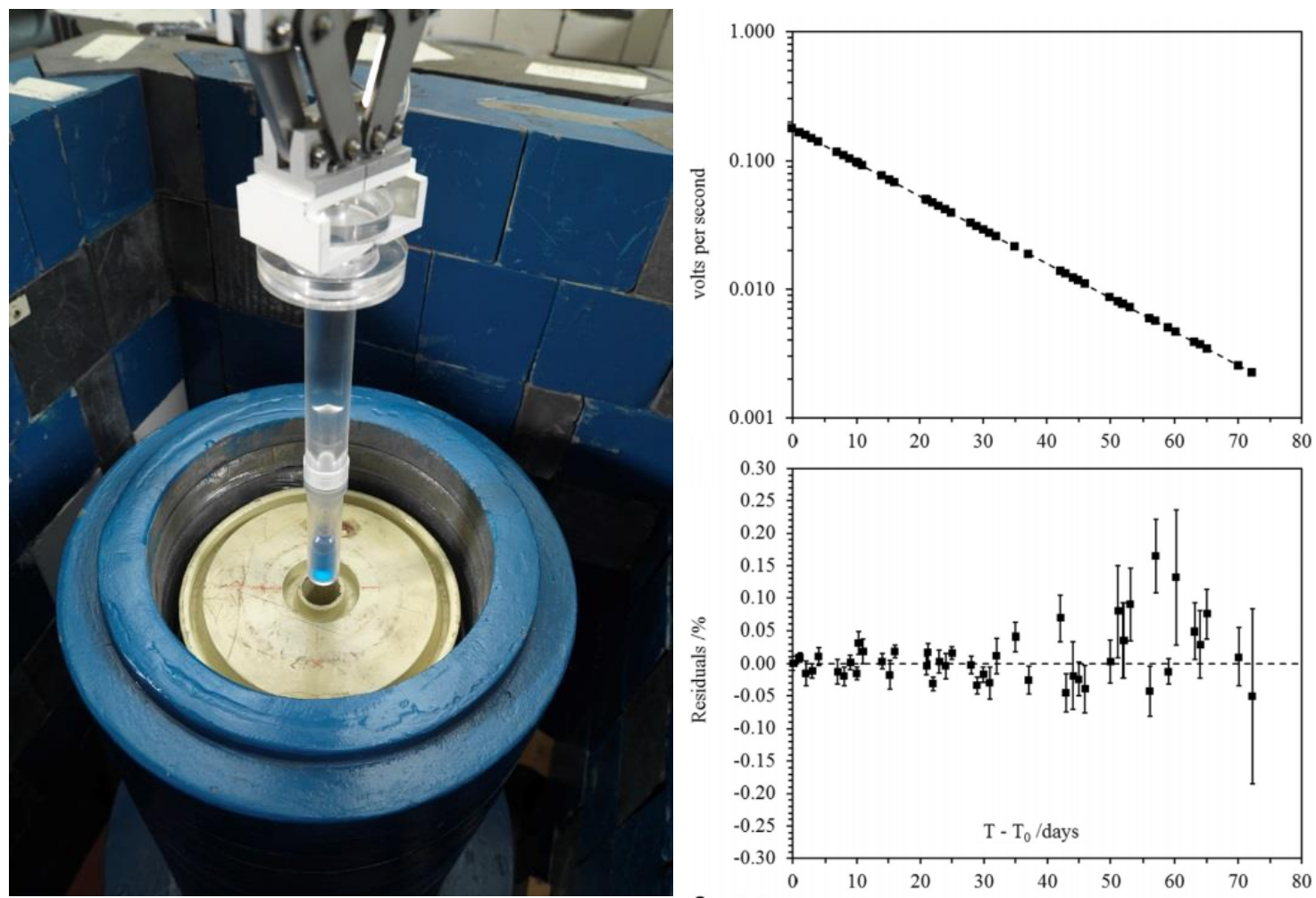

Figure 3 (Left) NPL ionization chamber with ampoule of radioactive material entering the well; (Right) Activity time distribution and half-life fits for the decay of ${ }^{223}$ Ra obtained using this ionization chamber [11].

Gamma-ray spectroscopy may be used as a secondary transfer method and also provides precision nuclear decay data. Hyper-pure germanium detectors can be used to measure any sample type and are isotopically selective, enabling measurement with more than one radionuclide present. If the specific activity $(A)$ is pre-determined by a primary standard technique, a precise full-energy peak detection efficiency for a gamma-ray detection system can be established from the expression,

$$
A=\frac{N}{P_{\gamma} \varepsilon_{\gamma} t}
$$


where $N$ is the background subtracted peak area, $t$ is the measurement time (which should be small relative to the decay half-life), $P_{\gamma}$ is the gamma emission probability per decay and $\varepsilon_{\gamma}$ is the absolute full-energy peak detection efficiency $\varepsilon_{\gamma}$. Since the provision of independent primary standards allows accurate determination of fullenergy peak detection efficiencies, such measurements can establish very precise $P_{\gamma}$ values $[12,13]$ which are used in subsequent secondary calibration sources (see figure $4)$. 



Figure 4: Absolute full-energy peak detection efficiency calibration curve and residuals for the LOKI HPGe detector at NPL used for absolute gamma-ray emission probability measurements of the decay of ${ }^{153} \mathrm{Gd}$, adapted from reference [13]. 


\section{Ongoing Applications of Standards.}

The application of radioisotopes for combined, parallel diagnostic and therapeutic use is another growth area where different isotopes of the same element which possess identical chemical properties (such as ${ }^{44} \mathrm{Sc} /{ }^{47} \mathrm{Sc},{ }^{64} \mathrm{Cu} /{ }^{67} \mathrm{Cu}$ and ${ }_{152,155} \mathrm{~Tb} / 149,161 \mathrm{~Tb}$ ) are particularly useful [14]. Radionuclide standards and reference materials are also required to determine the activity from environmental sources. Public confidence in all nuclear pursuits stems from traceable measurement, underpinned by precise and evaluated nuclear decay data. Such measurements are essential for applications including environmental radioactivity monitoring and decommissioning, verifying compliance with the Comprehensive Test Ban Treaty and within nuclear medicine. Specifically, there are a number of emerging technologies in the radiopharmaceutical arena which require novel and challenging primary standardizations before they can be exploited in a clinical scenario. Each application has an impact in connecting nuclear science measurements with societal exploitation through the dissemination of results from nuclear physics research. Simply put, without traceable standards there can be no confidence in any radiation measurement.

\section{Acknowledgements}

This work is supported via the UK Department for Business, Energy and Industrial Strategy and the Science and Technologies Facility Council. The authors grateful for discussions with S.M. Collins and R.Shearman. 


\section{$\underline{\text { References }}$}

[1] E. Rutherford, Radium Standards and Nomenclature, Nature, vol. 84, No. 2136 (1910) p430-431

[2] E.A.McCutchan, D.A.Brown and A.A.Sonzogni, A new look at Nuclear Data, Nuclear Physics News , vol 27, number 3 (2017) p5-9 ; DDEP: http://www.nucleide.org/DDEP WG/DDEPdata.htm

[3] S. Pomme, Methods for primary standardization of activity, Metrologia, vol. 44, (2007) p17-26 http://dx.doi.org/10.1088/0026-1394/44/4/S03

[4] S.M.Judge, D. Arnold, B. Chauvenet et al., 100 Years of Radionuclide Metrology, Applied Radiation and Isotopes vol. 87 (2014) p27-31 https://doi.org/10.1016/j.apradiso.2013.11.121

[5] Ryszard Broda, Phillipe Cassette and Karsten Kossert, Radionuclide metrology using liquid scintillation counting," Metrologia, vol. 44, no. 4 (2007) S36-S52. http://dx.doi.org/10.1088/026-1394/44/4/S06

[6] B.E.Zimmerman, D.E.Bergon, J.T.Cessna et al., Revision of the NIST Standard for ${ }^{223}$ Ra: New Measurements and Review of 2008 Data, Journal of Research of the National Institute of Standards and Technology Vol. 120 (2015) 37-57 http://dx.doi.org/10.6028/ires.120.004; John Keightley, Andy Pearce, Andrew Fenwick et al., Standardisation of ${ }^{223}$ Ra by liquid scintillation counting and comparison with secondary measurements, Applied Radiation and Isotopes vol. 95 (2015) p114-121 https://doi.org/10.1016/j.apradiso.2014.10.009 ;

[7] Karsten Kossert, Karen Bokeloh, Rainer Dersch et al., Activity determination of ${ }^{227} \mathrm{Ac}$ and ${ }^{223} \mathrm{Ra}$ by means of liquid scintillation counting and determination of nuclear decay data, Applied Radiation and Isotopes vol. 95 (2015) p143-152. https://doi.org/10.1016/j.apradiso.2014.10.005

[8] S. Pommé The uncertainty of counting at a defined solid angle Metrologia vol. 52 (2015) S73-85 http://dx.doi.org/10.1088/0026-1394/52/3/S73

[9] Claude J. Bailat et al. International Comparison CCRI(II)-S7 on the analysis of Uncertainty Budgets for $4 \pi \beta \gamma$ Coincidence Counting Metrologia 51 (2014) Tech. Suppl. 06018 https://doi.org/10.1088/0026-1394/51/1A/06018

[10] S.M.Collins, R.Shearman, J.D.Keightley and P.H.Regan, Investigation of $\gamma-\gamma$ coincidence counting using the National Nuclear Array (NANA) as a primary standard, Applied Radiation and Isotopes, vol. 134 (2018) p290-296, https://doi.org/10.1016/j.apradiso.2017.07.056

[11] S.M.Collins, A.K.Pearce, K.M.Ferreira et al., Direct measurement of the half-life of ${ }^{223}$ Ra Applied Radiation and Isotopes, vol. 99 (2015) p46-53 https://doi.org/10.1016/j.apradiso.2015.02.003 
[12] S.M.Collins, A.K.Pearce, P.H.Regan \& J.D.Keightley, Precise measurements of the absolute $y$-ray emission probabilities of ${ }^{223} \mathrm{Ra}$ and decay progeny in equilibrium, Applied Radiation and Isotopes vol. 102 (2015) p15-28

https://doi.org/10.1016/j.apradiso.2015.04.008

[13] R.Shearman, S.M.Collins, J.D.Keightley, A.K.Pearce and J. Garnier, Absolute intensities of the gamma-ray emissions originating from the electron capture decay of ${ }^{153} \mathrm{Gd}$, European Physical Journal Web of Conferences 146,10008 (2017), https://doi.org/10.1051/epjconf/201714610008

[14] Cristina Muller, Nicholas P. Van de Meulen Martina Benesova and Roger Schibli, Therapeutic Radiometals Beyond ${ }^{177} \mathrm{Lu}$ and ${ }^{90} \mathrm{Y}$ : Production and Application of Promising $\alpha$-Particle, $\beta$-Particle, and Auger Electron Emitters, J Nucl Med 58 (2017) 91S-96S, https://doi.org/10.2967/jnumed.116.186825 


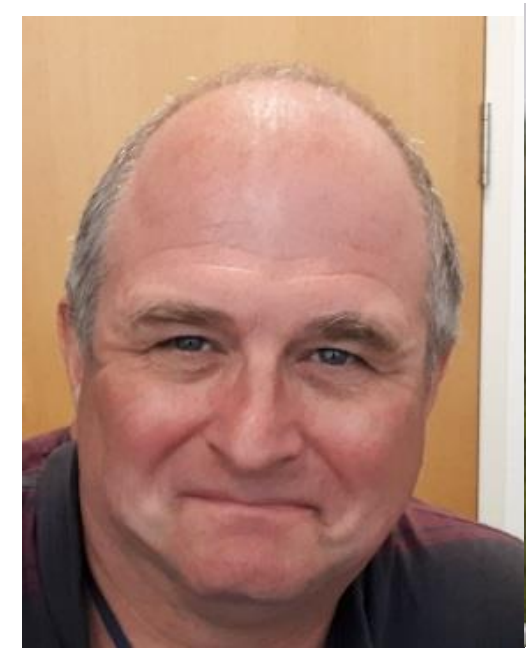

Patrick Regan

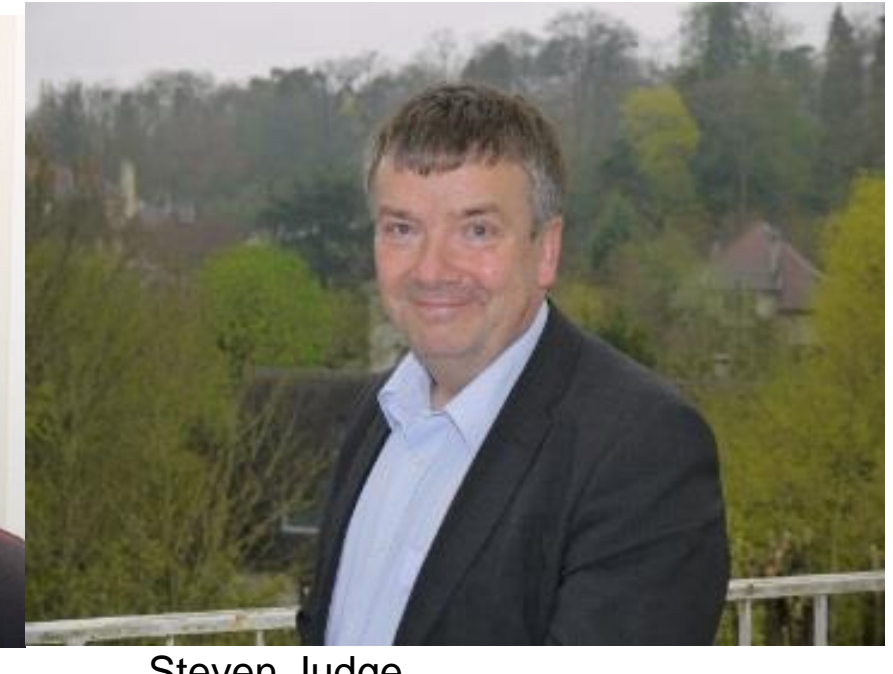

Steven Judge

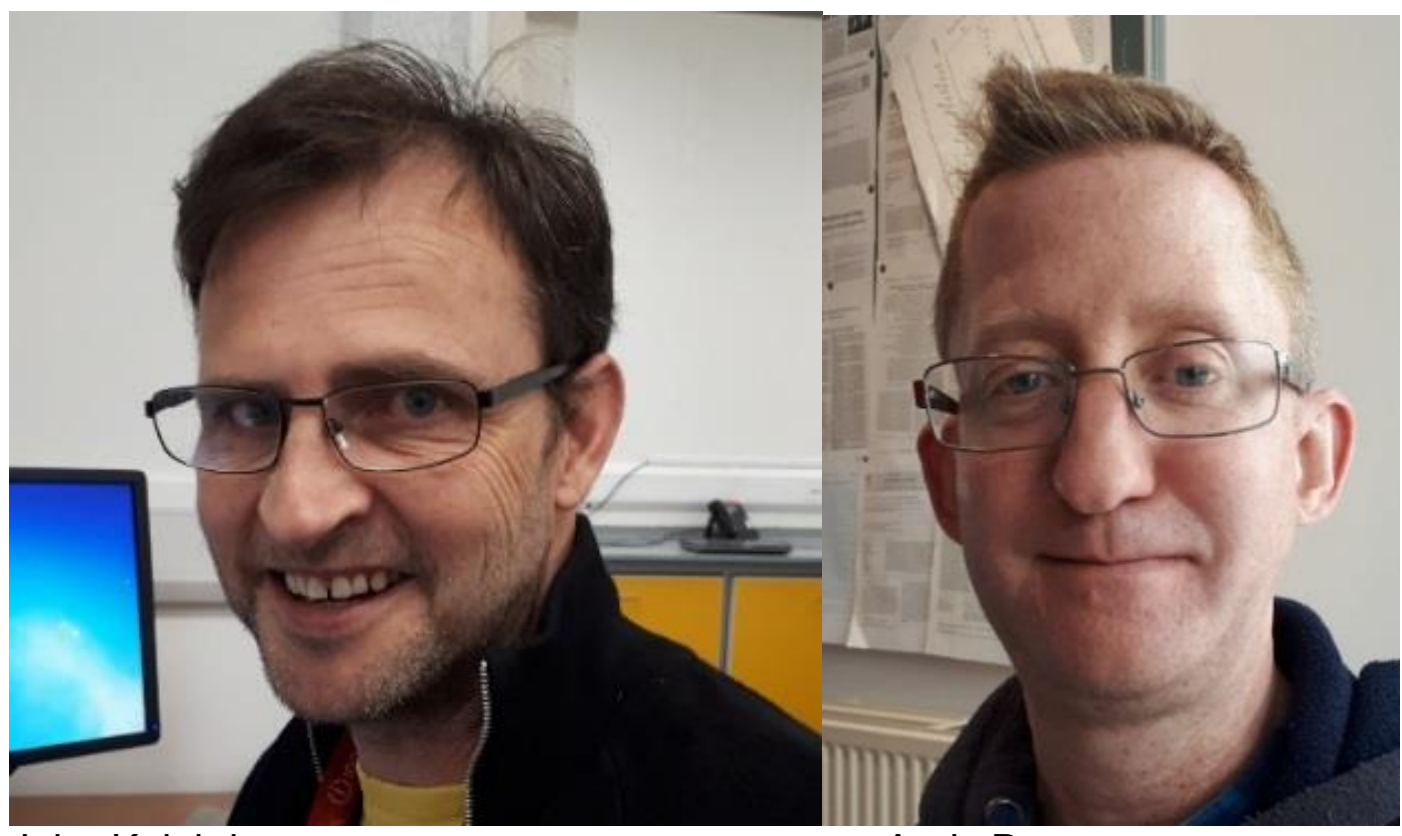

John Keightley

Andy Pearce 
SEPARATE FIGURE PAGES:

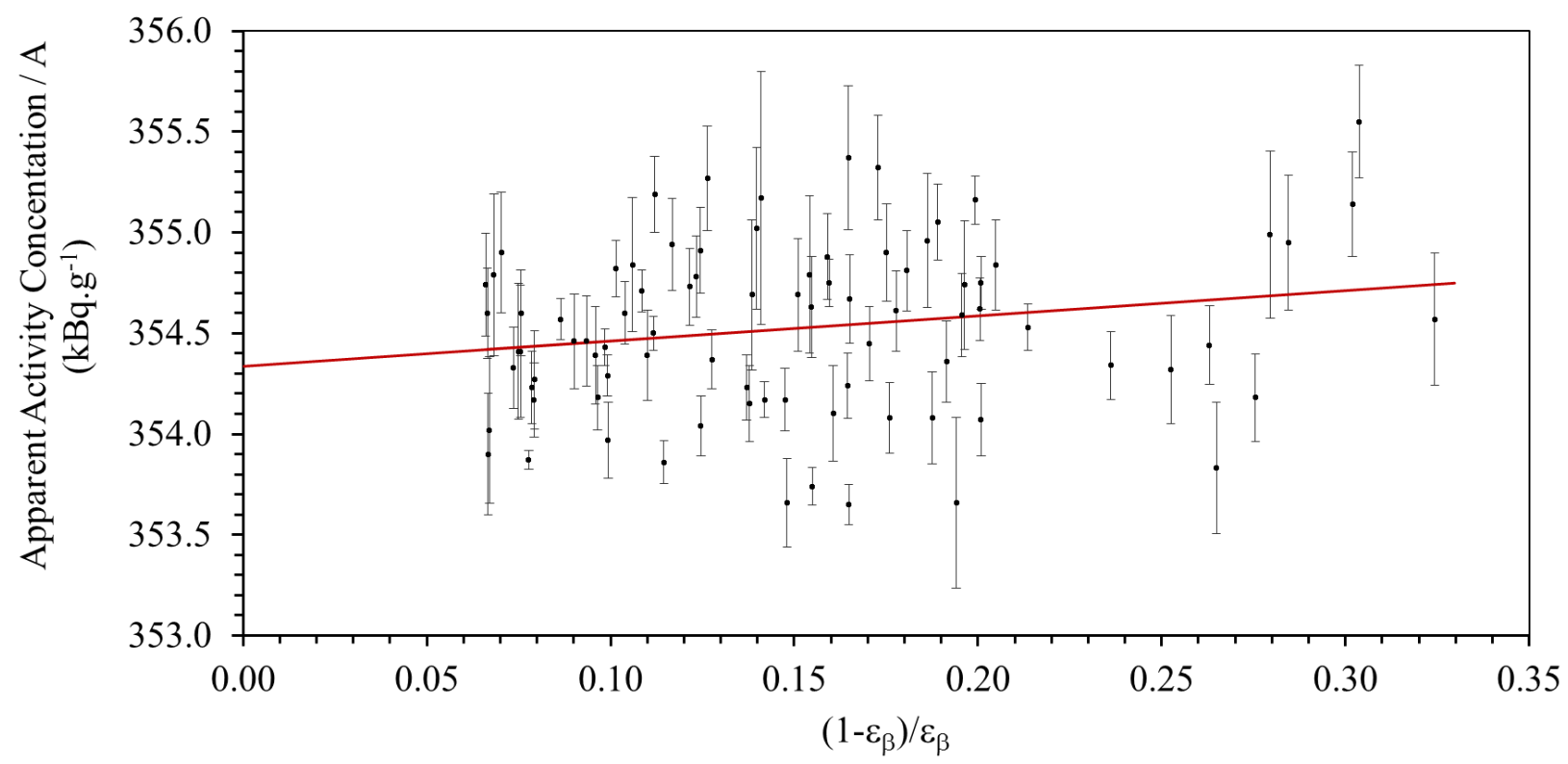

Figure 1 Efficiency extrapolation curve for ${ }^{60} \mathrm{Co}$ based on the $1173 \mathrm{keV}$ and $1332 \mathrm{keV}$ gamma full-energy peak coincidences with the $4 \pi$ atmospheric pressure proportional counter at the UK's National Physical Laboratory, figure adapted from ref. [9]. 

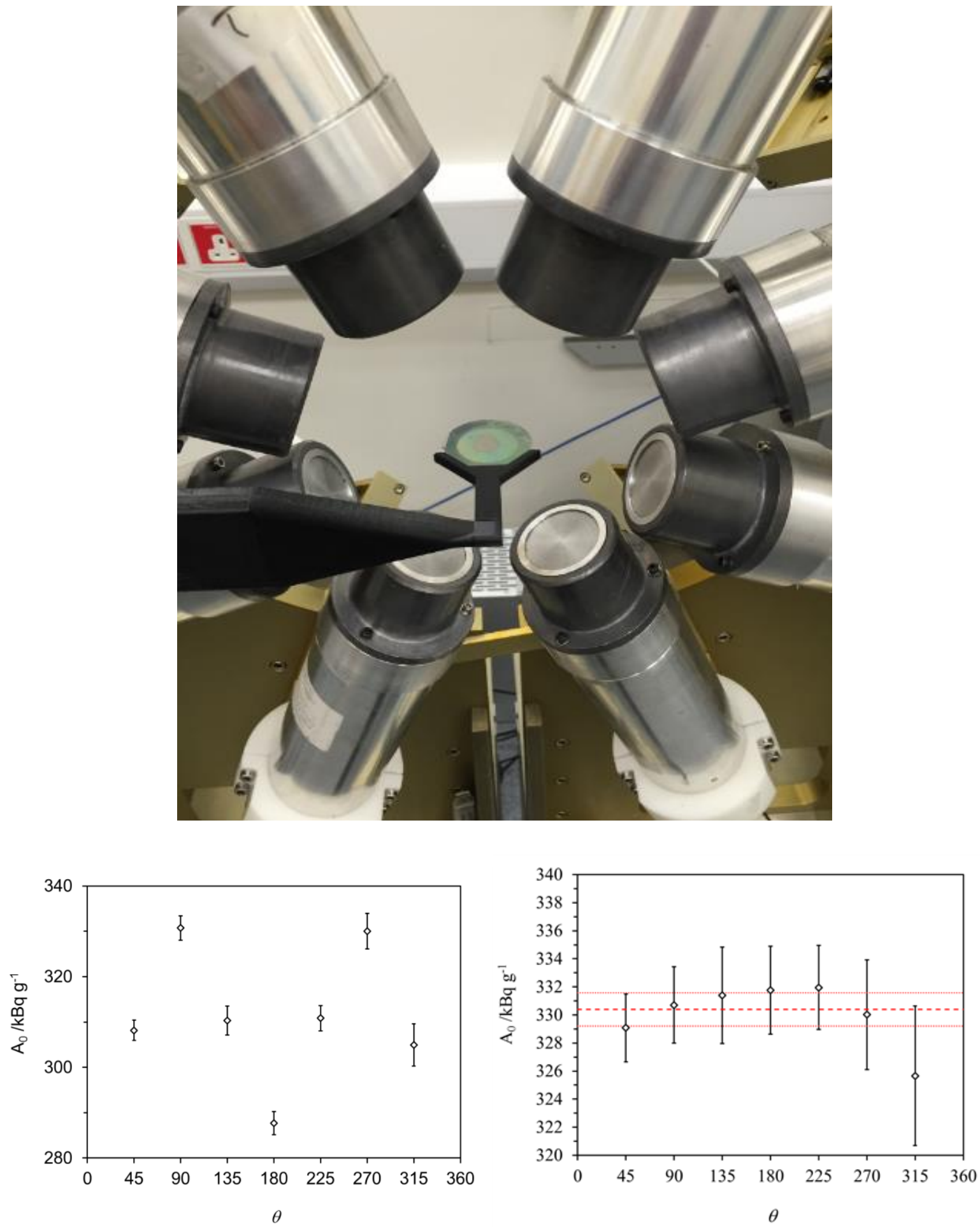

Figure 5: Upper: The UK National Nuclear Array (NANA) which can be used for primary standardisations using the gamma-ray coincidence method; Lower: example of raw and angular correlation corrected primary activity values for $a^{60} \mathrm{Co}$ standard taken using NANA [10]. 

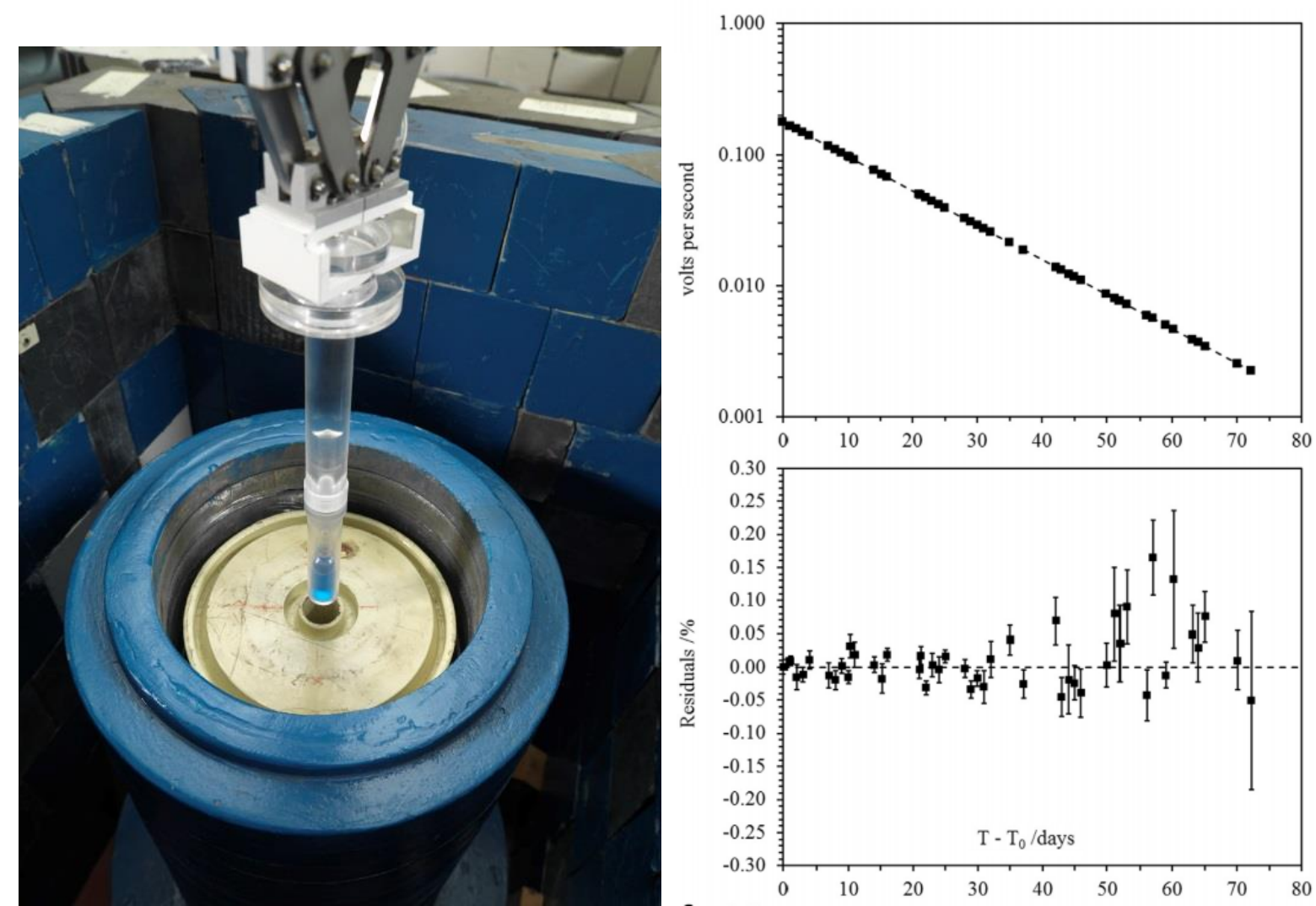

Figure 3: (Left) NPL ionization chamber with ampoule of radioactive material entering the well; (Right) Activity time distribution and half-life fits for the decay of ${ }^{223}$ Ra obtained using this ionization chamber [11]. 

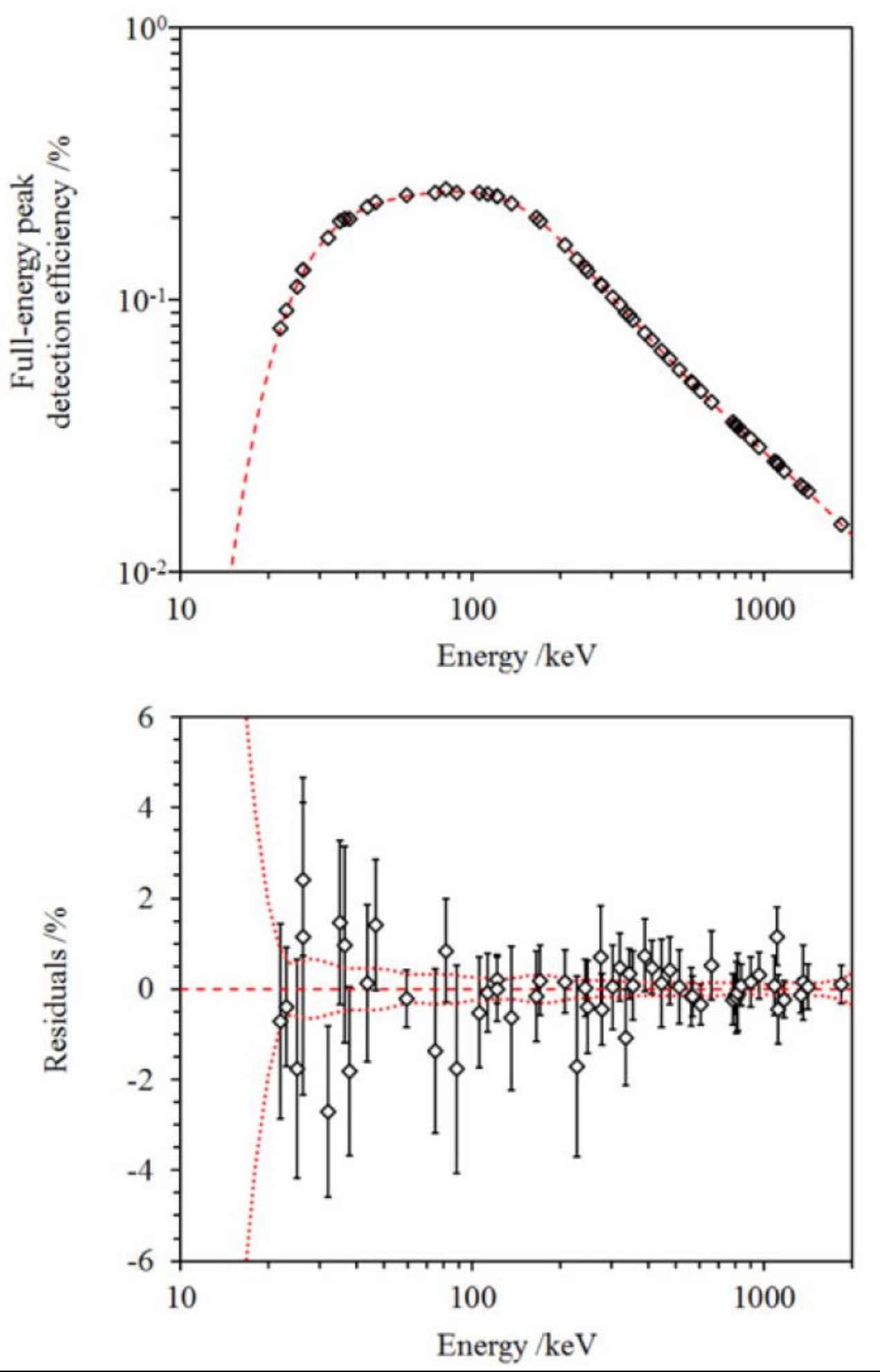

Figure 4: Absolute full-energy peak detection efficiency calibration curve and residuals for the LOKI HPGe detector at NPL used for absolute gamma-ray emission probability measurements of the decay of ${ }^{153} \mathrm{Gd}$, adapted from reference [13]. 